\section{Hospitalizações e óbitos relacionados à esporotricose no Brasil (1992-2015)}

\author{
Hospitalizations and deaths related to \\ sporotrichosis in Brazil (1992-2015)
}

\section{Hospitalizaciones y óbitos relacionados con la esporotricosis en Brasil (1992-2015)}

\section{Resumo}

A esporotricose é uma micose subcutânea de distribuição global e, em geral, os pacientes são tratados ambulatorialmente. Desde 1998, observa-se aumento dos casos no Estado do Rio de Janeiro, Brasil, especialmente por transmissão zoonótica envolvendo gatos. Os pacientes coinfectados pelo vírus da imunodeficiência humana (HIV), muitas vezes, necessitam de hospitalizações e evoluem a óbito. Este estudo analisa e descreve dados de 1992 a 2015, provenientes de bancos de dados do Sistema Único de Saúde. No Brasil, ocorreram 782 hospitalizações e 65 óbitos. Em 6\% das hospitalizações e 40\% dos óbitos, havia coinfecção pelo HIV. No Rio de Janeiro, foram 250 hospitalizações e 36 óbitos, com aumento progressivo, ao longo do período. Destacaram-se, ainda, São Paulo e Goiás. Homens, não brancos, com baixa escolaridade evoluíram mais frequentemente a óbito. Conclui-se que a esporotricose está associada a hospitalizações é óbitos em todo o Brasil, com destaque para o Rio de Janeiro.

Esporotricose; Micoses; HIV; Hospitalização; Registros de Mortalidade
COMUNICAÇÃO BREVE

BRIEF COMMUNICATION

\author{
Eduardo Mastrangelo Marinho Falcão 1 \\ José Berilo de Lima Filho 2 \\ Dayse Pereira Campos † \\ Antonio Carlos Francesconi do Valle 2 \\ Francisco Inácio Bastos 3 \\ Maria Clara Gutierrez-Galhardo 2 \\ Dayvison Francis Saraiva Freitas 1,2
}

doi: 10.1590/0102-311X00109218

\author{
Correspondência \\ E. M. M. Falcão \\ Instituto Oswaldo Cruz, Fundação Oswaldo Cruz. \\ Av. Brasil 4365, Rio de Janeiro, RJ 21040-360, Brasil. \\ edummf@yahoo.com.br \\ 1 Instituto Oswaldo Cruz, Fundação Oswaldo Cruz, Rio de \\ Janeiro, Brasil. \\ 2 Instituto Nacional de Infectologia Evandro Chagas, Fundação \\ Oswaldo Cruz, Rio de Janeiro, Brasil. \\ 3 Instituto de Comunicação e Informação Científica e Tecnologia \\ em Saúde, Fundação Oswaldo Cruz, Rio de Janeiro, Brasil. \\ + Falecida.
}




\section{Introdução}

A esporotricose, micose subcutânea mais frequente em países de clima quente e úmido, tem sua transmissão classicamente descrita por inoculação traumática com plantas ou solo com fungos do gênero Sporothrix. Entretanto, atualmente, é notável a transmissão zoonótica por mordedura, arranhadura ou contato direto com gatos infectados ${ }^{1}$. As formas cutâneas localizadas, de evolução benigna, predominam. Raramente, ocorre disseminação e acometimento extracutâneo, por vezes fatal, em geral, em pacientes com condições imunossupressoras como aids e etilismo 2 .

Surtos têm sido descritos em todo o mundo, observando-se amplo espectro clínico ${ }^{3}$, sendo o maior deles registrado na África do Sul, com acometimento de mais de 3 mil trabalhadores de minas de ouro, 5 com formas graves 4 . No principal surto dos Estados Unidos, envolvendo 84 pacientes, 20\% foram hospitalizados. A letalidade hospitalar foi de 3\% e a incidência de hospitalizações de 0,35 por milhão de habitantes/ano, no período 2000-2013 5.

Desde 1998, ocorre uma hiperendemia de esporotricose no Estado do Rio de Janeiro, de transmissão zoonótica pelo gato 6, associada a Sporothrix brasiliensis, espécie mais virulenta do gênero 7 . Nota-se, com o avanço da hiperendemia e acometimento de indivíduos vulneráveis, incremento na morbimortalidade 2 .

No Brasil, a esporotricose é de notificação compulsória no Rio de Janeiro, Pernambuco, Paraíba e municípios de Guarulhos (São Paulo), Camaçari (Bahia) e Conselheiro Lafaiete (Minas Gerais). Com relatos e séries de casos concentrados no Sul e Sudeste, não se conhece o impacto da morbimortalidade nacional. Objetivou-se analisar a distribuição da esporotricose pelas hospitalizações e óbitos no Brasil, utilizando dados do sistema único de saúde.

\section{Metodologia}

Analisaram-se dados de domínio público do Sistema de Informações Hospitalares do Sistema Único de Saúde (SIH-SUS) e do Sistema de Informações sobre Mortalidade (SIM), de 1992 a 2015. O estudo foi aprovado pelo Comitê de Ética em Pesquisa do Instituto Nacional de Infectologia Evandro Chagas (INI), Fundação Oswaldo Cruz (Fiocruz) - CAAE 61114616.4.0000.5262. Foram incluídos registros com códigos de esporotricose da Classificação Internacional de Doenças (CID) e utilizados dados dos censos e estimativas populacionais do Instituto Brasileiro de Geografia e Estatística (IBGE) para calcular a taxa de hospitalizações.

\section{Resultados}

Entre 1992 e 2015, no Brasil, a esporotricose foi registrada em 782 hospitalizações (Figura 1), em todos os estados (exceto Roraima), com duração mediana de 8 dias, mediana de idade de 43 anos e hospitalização mais longa entre os homens ( $\mathrm{p}<0,0001$; Tabela 1).

Os estados com maior número de hospitalizações foram Rio de Janeiro, com 250 (32\%), São Paulo, 128 (16,4\%) e Goiás, 69 (8,8\%). A taxa de hospitalizações por milhão de habitantes no Brasil foi de 0,11, em 1992, e 0,23, em 2015, com oscilações relevantes. No Rio de Janeiro, foi de 0,85, em 1992, e 1,08, em 2015, mantendo-se acima de 1,03 a partir de 2013. Em São Paulo, foi de 0,03, em 1992, e 0,13, em 2015. Já em Goiás, foi de 0,24, em 1992, entre 2008 e 2011, manteve-se acima de 2,31 (3,03 em 2011), mas 0,00 em 2015.

Houve picos das taxas registradas na Bahia (1,36, em 1994), Tocantins (1,61, em 2002, mantendose acima de 0,77 até 2004), Acre (1,41, em 2008), Paraná (1,04, em 2008), Amazonas (5,09, em 2009), Distrito Federal (1,57 e 1,05, em 2009 e 2014), Amapá (1,42 em 2011) e Paraíba (1,01 em 2014).

Entre 1998 e 2015, período com menção do município da hospitalização, foram registradas 682 (87,2\% do total) hospitalizações em 302 municípios (5,4\% dos municípios do Brasil). Rio de Janeiro e Duque de Caxias lideraram (Figura 2). Em 612 (89,7\%), esporotricose foi o diagnóstico primário, sendo 220 (35,9\%) casos pela forma pulmonar, 129 (21,1\%), linfocutânea, 76 (12,4\%), disseminada, $70(11,4 \%)$, outras formas de esporotricose e $117(19,1 \%)$ por esporotricose não especificada. 


\section{Figura 1}

Hospitalizações e óbitos relacionados à esporotricose no Brasil, no período de 1992 a 2015.

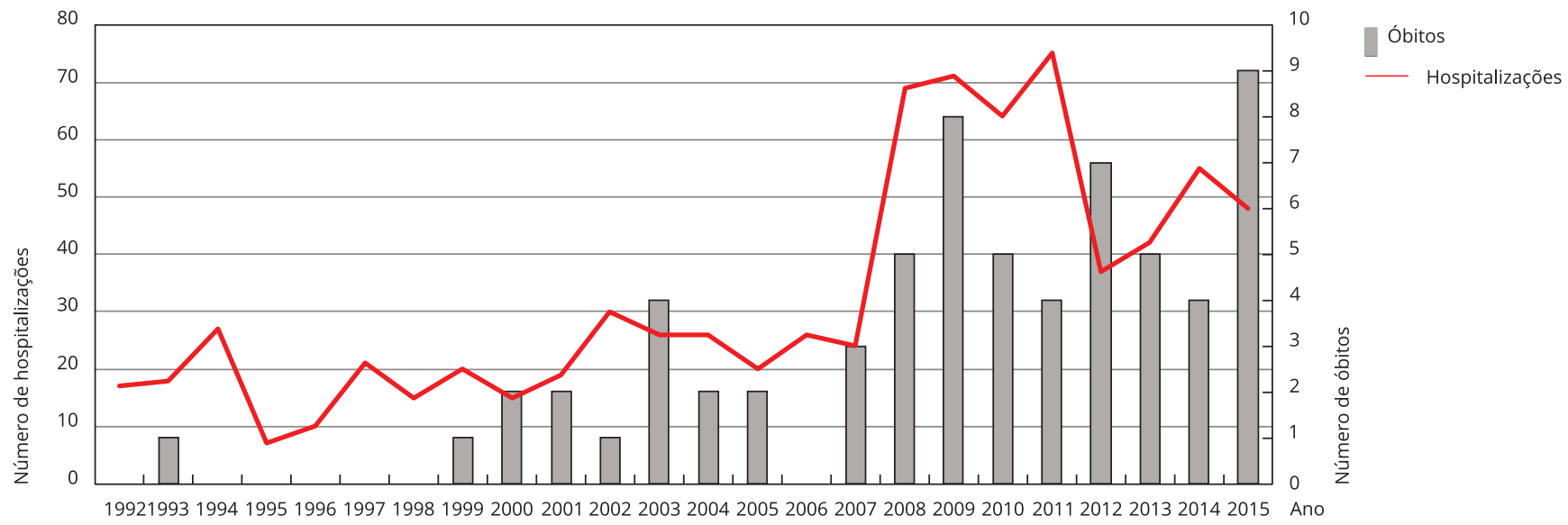

Fonte: Sistema de Informações Hospitalares do Sistema Único de Saúde (SIH-SUS) e Sistema de Informações sobre Mortalidade (SIM), 2017.

\section{Tabela 1}

Características sociodemográficas das hospitalizações e óbitos relacionados à esporotricose no Brasil, de 1992 a 2015.

\begin{tabular}{|c|c|c|c|c|}
\hline \multirow[t]{2}{*}{ Características } & \multicolumn{3}{|c|}{ Hospitalizações } & \multirow{2}{*}{$\begin{array}{c}\text { Óbitos } \\
\text { n (\%) }\end{array}$} \\
\hline & n (\%) & Duração mediana (dias) & Valor de p & \\
\hline Gênero & & & $<0,0001$ * & \\
\hline Masculino & $474(60,6)$ & 9,0 & & $45(69,2)$ \\
\hline Feminino & $303(38,7)$ & 7,0 & & $20(30,8)$ \\
\hline Faixa etária (anos) & & & $<0,0001 * *$ & \\
\hline $0-24$ & $195(24,9)$ & 5,0 & & $4(6,2)$ \\
\hline $25-60$ & $386(49,3)$ & 9,0 & & $46(70,7)$ \\
\hline$>60$ & $196(25,1)$ & 9,0 & & $15(23,1)$ \\
\hline Cor da pele & & & - & \\
\hline Não branca & - & - & & $40(61,5)$ \\
\hline Branca & - & - & & $22(33,8)$ \\
\hline Indisponível & - & - & & $3(4,6)$ \\
\hline Escolaridade (anos) & & & - & \\
\hline Até 7 & - & - & & $37(56,9)$ \\
\hline$>7$ & - & - & & $10(15,4)$ \\
\hline Indisponível & - & - & & $18(27,7)$ \\
\hline \multicolumn{5}{|l|}{$\mathrm{HIV+}$} \\
\hline Sim & - & - & & $26(40,0)$ \\
\hline Não & - & - & & $39(60,0)$ \\
\hline
\end{tabular}

Fonte: Sistema de Informações Hospitalares do Sistema Único de Saúde (SIH-SUS) e Sistema de Informações sobre Mortalidade (SIM), 2017.

Nota: as variáveis Gênero e Faixa etária estavam indisponíveis em 5 (0,6\%) registros, respectivamente.

* Teste de Wilcoxon-Mann-Whitney;

** Teste de Kruskal-Wallis. 


\section{Figura 2}

Hospitalizações relacionadas à esporotricose no Brasil, por município de residência, no período de 1998 a 2015.

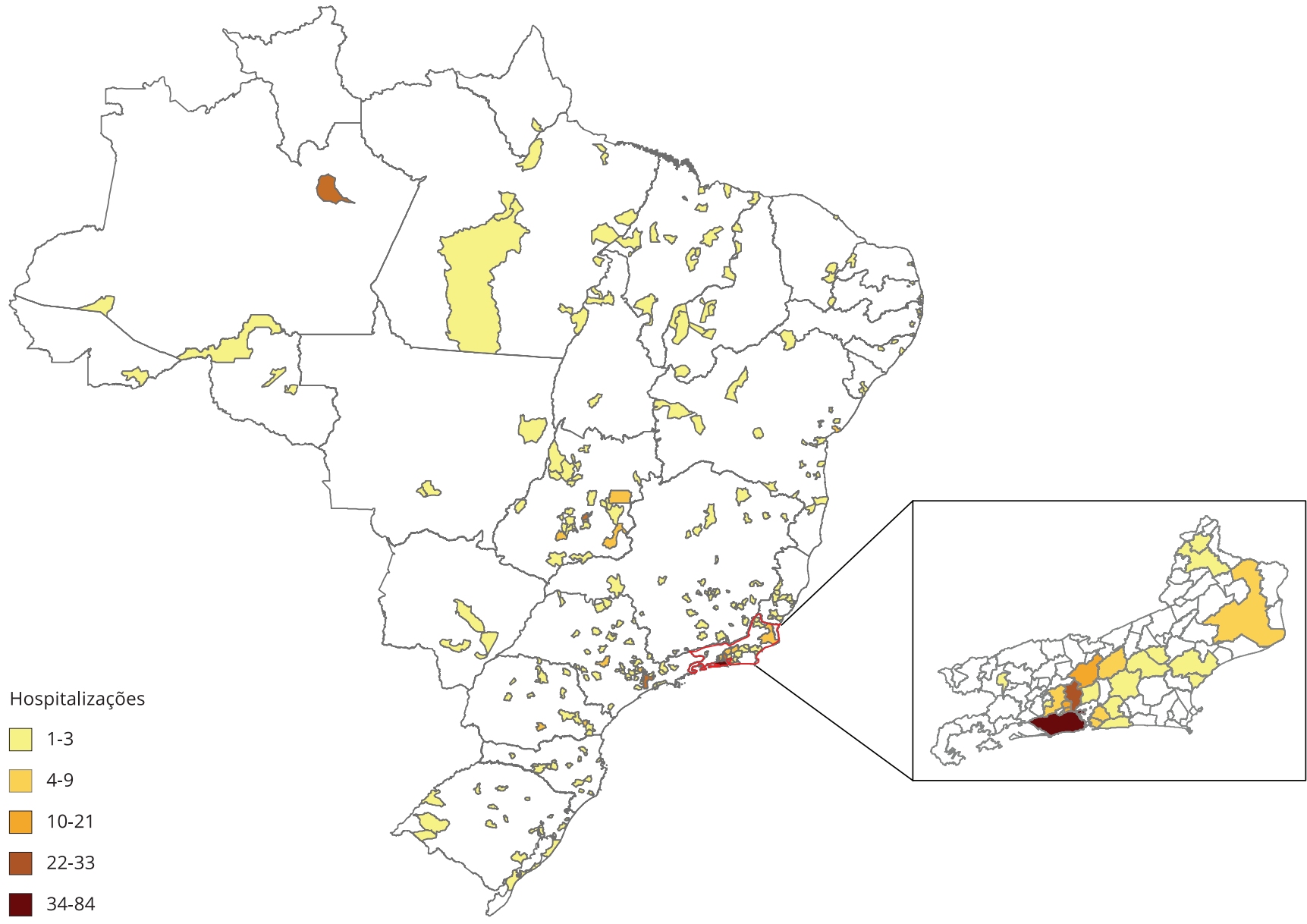

Fonte: Sistema de Informações Hospitalares do Sistema Único de Saúde (SIH-SUS) e Sistema de Informações sobre Mortalidade (SIM), 2017.

O diagnóstico secundário foi informado em 22,8\% do total: doença pelo HIV (5), neoplasias malignas (4), transplante hepático (2), etilismo (2) e agranulocitose (1); doenças crônico-degenerativas (4); doenças infecciosas (10). A esporotricose foi informada como diagnóstico secundário em 11,7\%, sendo em 36 hospitalizações $(5,3 \%)$ o diagnóstico primário de doença pelo HIV.

Entre 1992 e 2015, a esporotricose foi reportada em 65 óbitos (Figura 1; Tabela 1), em 10 (37\%) das Unidades Federativas brasileiras, por local de residência: Rio de Janeiro, 36 (55,4\%), Minas Gerais, 12 (18,5\%), São Paulo, 6 (9,2\%), Rio Grande do Sul, 4 (6,2\%), Paraná, 2 (3,1\%) e Bahia, Goiás, Pará, Santa Catarina e Sergipe com 1 óbito cada um. O município com mais óbitos foi o Rio de Janeiro (15; 23,1\%). A instituição com maior número de óbitos foi o INI/Fiocruz (9; 13,8\%).

A forma clínica disseminada foi reportada em 8 (12,3\%) casos, seguida de linfocutânea, pulmonar e outras formas de esporotricose, com 3 (4,6\%) casos cada uma. Esporotricose não especificada foi registrada em $48(73,8 \%)$ casos.

Dentre os diagnósticos relacionados diretamente ao óbito, destacaram-se: doença pelo HIV, em 21 (32,3\%), desnutrição, em 2 (3\%), etilismo, imunodeficiência não especificada, lúpus eritematoso, linfoma de Hodgkin, leucemia mieloide aguda, em 1, cada (total de 7,7\%) uma. Também foram regis- 
tradas doenças/agravos degenerativos e infecções. HIV foi ainda informado como comorbidade, mas sem determinar óbito, em 5 (7,6\%).

Quando considerados os 26 (40\% do total) óbitos de pacientes com HIV, a idade mediana foi 36 anos; $76,9 \%$ eram do sexo masculino, $73 \%$, não brancos e 50\% estudaram menos que 7 anos.

\section{Discussão}

O estudo produziu informações sobre uma doença emergente, classicamente de baixa gravidade, com raro acometimento extracutâneo 1, mas está associada a hospitalizações e óbitos. Contudo, esses dados podem ser ainda maiores, uma vez que a esporotricose não é uma doença de notificação compulsória em todo o país.

Os dados são compatíveis com a hiperendemia por transmissão zoonótica no Rio de Janeiro, sua concentração na região metropolitana e o aumento do número dos casos graves, com atendimento de referência centrado no INI/Fiocruz, desde os primórdios da hiperendemia 1,2,6.

É provável que o aumento do número de casos desde 2010, em São Paulo, também esteja associado à transmissão zoonótica, considerando o aumento no número de casos de esporotricose felina 8 .

Goiás se destacou pelo número de hospitalizações no período, sendo mais da metade (53\%) na região metropolitana de Goiânia, apesar de não existirem relatos de surtos de esporotricose na literatura ou nos bancos de informações de vigilância em saúde do estado. Sobreira et al. ${ }^{9}$ descreveram, em 2016, em congresso de medicina tropical, sete casos de esporotricose em felinos, no Município de Anápolis, a 50km de Goiânia. É provável a ocorrência de surto da doença, porém são necessários estudos detalhados para esclarecimento das fontes de contágio e do número de infectados.

A maior taxa de hospitalização no período foi registrada no Amazonas, em 2009, onde a esporotricose já foi a micose subcutânea predominante. Os períodos de aumento de hospitalizações na Bahia e no Paraná poderiam estar associados aos casos de esporotricose felina 8 . Assim como Goiás, merecem investigações Tocantins, Acre, Amapá e Paraíba, pelos picos de hospitalizações.

Surpreende a principal forma de esporotricose associada à hospitalização ser a pulmonar. Na literatura, há raros casos dessa forma 1, corroborando o observado no INI/Fiocruz. Como primeiro código listado (B42.0), pode ter ocorrido equívoco no preenchimento dessa forma clínica, ao invés de sua ocorrência real.

Entre os óbitos, o predomínio da forma disseminada ratifica sua maior mortalidade por quadros graves, com acometimento de órgãos vitais, como o sistema nervoso central 1,2.

Além da infecção pelo HIV, etilismo, desnutrição e outras condições imunossupressoras, registradas como causas secundárias nas hospitalizações e óbitos, parecem tornar esses grupos mais vulneráveis a quadros graves.

Houve maior duração de hospitalizações em pacientes do sexo masculino. Os óbitos predominaram em homens não brancos com baixa escolaridade, sugerindo um perfil da população vulnerável, exposta ao risco de doença mais grave. Em estudos realizados na região hiperendêmica do Rio de Janeiro, mulheres são as mais acometidas 1,6, enquanto os casos mais graves ocorrem em homens, provavelmente em razão da sobreposição de endemias (HIV/aids e esporotricose) 2.

Pressupõe-se uma letalidade hospitalar próxima de 8,3\%, superior à relatada nos Estados Unidos 5 , sugerindo que a hospitalização no Brasil envolva casos mais graves. Os bancos de dados do SIH-SUS e SIM são ferramentas valiosas na vigilância epidemiológica no país 10 e permitiram, neste estudo, a identificação dos casos graves e fatais, chamando a atenção para tais desfechos. Porém, a não integração dos bancos e a inexistência de um banco com os casos de esporotricose foram obstáculos para a exatidão dos dados.

Conclui-se que a esporotricose assume um perfil mais grave nacionalmente. O Rio de Janeiro apresenta perfil clínico-epidemiológico de transmissão zoonótica especialmente grave, no entanto alertamos para a ocorrência ainda pouco visível em outros estados. Além disso, o maior número de óbitos em uma população desfavorecida expõe a questão da desigualdade social, enquanto ampliação de vulnerabilidade e desigualdade de oportunidades. Sugere-se a inclusão da esporotricose na lista de agravos de notificação compulsória nacional, assim como a ampliação das medidas de vigilância e controle em todo o país. 


\section{Colaboradores}

E. M. M. Falcão contribuiu com a concepção e projeto, aquisição, análise e interpretação dos dados, e redação do artigo. J. B. Lima Filho contribuiu com a aquisição, análise e interpretação dos dados e revisão crítica do conteúdo intelectual. D. P. Campos contribuiu com a concepção e projeto, análise dos dados e revisão do conteúdo científico. A. C. F. Valle contribuiu com a concepção e projeto, análise e interpretação dos dados e revisão crítica do conteúdo intelectual. F. I. Bastos contribuiu com a análise e interpretação dos dados e revisão crítica do conteúdo intelectual. M. C. Gutierrez-Galhardo e D. F. S. Freitas contribuíram com a concepção e projeto, análise e interpretação dos dados, redação e revisão crítica do conteúdo intelectual.

\section{Informações adicionais}

ORCID: Eduardo Mastrangelo Marinho Falcão (0000-0003-2118-653X); José Berilo de Lima Filho (0000-0001-5338-9636); Antonio Carlos Francesconi do Valle (0000-0003-0890-4525); Francisco Inácio Bastos (0000-0001-5970-8896); Maria Clara Gutierrez-Galhardo (0000-0001-7906-8275); Dayvison Francis Saraiva Freitas (0000-0003-20392438).

\section{Agradecimentos}

Anselmo Rocha Romão (Instituto de Comunicação e Informação Científica e Tecnologia em Saúde, Fundação Oswaldo Cruz) pelo auxílio na confecção do mapa da Figura 2.

\section{Referências}

1. Barros MB, Almeida-Paes R, Schubach AO. Sporothrix schenckii and sporotrichosis. Clin Microbiol Rev 2011; 24:633-54.

2. Freitas DF, do Valle AC, da Silva MBT, Campos DP, Lyra MR, de Souza RV, et al. Sporotrichosis: an emerging neglected opportunistic infection in HIV-infected patients in Rio de Janeiro, Brazil. PLoS Negl Trop Dis 2014; 8:e3110.

3. Chakrabarti A, Bonifaz A, Gutierrez-Galhardo MC, Mochizuki T, Li S. Global epidemiology of sporotrichosis. Med Mycol 2015; 53:3-14.

4. Lurie HI. Five unusual cases of sporotrichosis from South Africa showing lesions in muscles, bones, and viscera. Br J Surg 1963; 50:585-91.

5. Gold JA, Derado G, Mody RK, Benedict K. Sporotrichosis-associated hospitalizations, United States, 2000-2013. Emerg Infect Dis 2016; 22:1817-20.

6. Barros MB, Schubach TM, Gutierrez-Galhardo MC, Schubach AO, Monteiro PC, Reis RS, et al. Sporotrichosis: an emergent zoonosis in Rio de Janeiro. Mem Inst Oswaldo Cruz 2001; 96:777-9.

7. Almeida-Paes R, de Oliveira MM, Freitas DF, do Valle AC, Zancopé-Oliveira RM, GutierrezGalhardo MC. Sporotrichosis in Rio de Janeiro, Brazil: Sporothrix brasiliensis is associated with atypical clinical presentations. PLoS Negl Trop Dis 2014; 8:e3094.

8. Gutierrez-Galhardo MC, Freitas DFS, do Valle ACF, Almeida-Paes R, Oliveira MME, Zancopé-Oliveira RM, et al. Epidemiological aspects of sporotrichosis epidemic in Brazil. Curr Fungal Infect Rep 2015; 9:238-45.

9. Sobreira EA, Ribeiro EL, Vasconcelos LSNOL, Braga CASB. Esporotricose felina: um problema de saúde pública detectado pelo Centro de Controle de Zoonoses de Anápolis-GO. In: Anais do Congresso da Sociedade Brasileira de Medicina Tropical (MEDTROP 2016). Maceió: Sociedade Brasileira de Medicina Tropical; 2016.

10. Lima CRA, Schramm JMA, Coeli CM, Silva MEM. Revisão das dimensões de qualidade dos dados e métodos aplicados na avaliação dos sistemas de informação em saúde. Cad Saúde Pública 2009; 25:2095-109. 


\section{Abstract}

Sporotrichosis is a subcutaneous mycosis with global distribution, and patients generally receive outpatient treatment. Since 1998 there has been an increase in cases in the state of Rio de Janeiro, Brazil, mainly via zoonotic transmission involving cats. Patients coinfected with the human immunodeficiency virus (HIV) often require hospitalization and evolve to death. This study analyzes and describes data from 1992 to 2015 obtained from the database of the Brazilian Unified National Health System (SUS). There were 782 hospitalizations and 65 deaths in Brazil. Six percent of the hospitalizations and $40 \%$ of the deaths involved coinfection with HIV. There were 250 hospitalizations and 36 deaths in Rio de Janeiro, with a progressive increase over the course of the period. The states of São Paulo and Goiás also showed high numbers. Men, non-whites, and individuals with low schooling evolved more frequently to death. In conclusion, sporotrichosis is associated with hospitalizations and deaths throughout Brazil, especially in the state of Rio de Janeiro.

Sporotrichosis; Mycoses; HIV; Hospitalization; Mortality Registries

\section{Resumen}

La esporotricosis es una micosis subcutánea de distribución global y, en general, los pacientes son tratados ambulatoriamente. Desde 1998, se observa un aumento de los casos en el estado de Río de Janeiro, Brasil, principalmente por transmisión zoonótica implicando gatos. Los pacientes coinfectados por el virus de la inmunodeficiencia humana (VIH), muchas veces, necesitan de hospitalizaciones y evolucionan a óbito. Este estudio analiza y describe datos de 1992 a 2015, provenientes de bancos de datos del Sistema Único de Salud (SUS). En Brasil, se produjeron 782 hospitalizaciones y 65 óbitos. En un 6\% de las hospitalizaciones y $40 \%$ de los óbitos, habia coinfección por el VIH. En Río de Janeiro, fueron 250 hospitalizaciones y 36 óbitos, con un aumento progresivo a lo largo del periodo. Se destacaron, incluso, São Paulo y Goiás. Hombres, no blancos, con baja escolaridad evolucionaron más frecuentemente a óbito. Se concluye que la esporotricosis está asociada a hospitalizaciones y óbitos en todo Brasil, destacando Río de Janeiro.

Esporotricosis; Micosis; VIH; Hospitalización; Registros de Mortalidad
Recebido em 05/Jun/2018

Versão final reapresentada em 13/Dez/2018

Aprovado em 13/Mar/2019 